\title{
Manipulating Robo Expression In Vivo Perturbs Commissural Axon Pathfinding in the Chick Spinal Cord
}

\author{
Stacey L. Reeber, ${ }^{1}$ Nozomi Sakai, ${ }^{1}$ Yuji Nakada, ${ }^{3}$ Judy Dumas, ${ }^{3}$ Kostantin Dobrenis, ${ }^{1}$ Jane E. Johnson, ${ }^{3}$ and \\ Zaven Kaprielian ${ }^{1,2}$ \\ ${ }^{1}$ Dominick P. Purpura Department of Neuroscience and ${ }^{2}$ Department of Pathology, Albert Einstein College of Medicine, Bronx, New York 10461, and \\ ${ }^{3}$ Department of Neuroscience, University of Texas Southwestern Medical Center, Dallas, Texas 75390
}

In vertebrate embryos, most spinal commissural axons cross the ventral midline (VM) and project either alongside or significant distances away from the floor plate (FP). The upregulation of repulsive Robo1/2 receptors on postcrossing commissural axons, in mammals, presumably allows these axons to respond to the midline-associated repellents, Slit1-3, facilitating their expulsion from, and prohibiting their reentry into, the FP. Compelling data suggest that Robo3 represses Robo1/2 function on precrossing axons and that Robo1/2 inhibit attractive guidance receptors on postcrossing axons, thereby ensuring that decussated axons are selectively responsive to midline Slits. However, whether Robo1/2 expel decussated commissural axons from the VM and/or prevent their reentry into the FP has not been explicitly established in vivo. Furthermore, some commissural axons do not require Robo1/2 to elaborate appropriate contralateral projections in the mouse spinal cord. Here, we use unilateral in ovo electroporation together with Atoh1 and Neurog1 enhancer elements to visualize, and assess the consequences of manipulating Robo expression on, dl1 and $\mathrm{dl} 2$ chick commissural axons. In response to misexpressing a cytoplasmic truncation of Robo1 and/or Robo2, which should block all Robo-ligand interactions, postcrossing commissural axons extend alongside, but do not project away from or reenter the FP. In contrast, misexpression of full-length Robo2 prevents many commissural axons from crossing the VM. Together, these findings support key and selective in vivo roles for Robo receptors in presumably altering the responsiveness of decussated commissural axons and facilitating their expulsion from the VM within the chick spinal cord.

Key words: Atoh1; Neurog1; floor plate; commissural axon; Robo; Slit

\section{Introduction}

Vertebrate commissural axons project along a transverse trajectory toward and across the floor plate (FP), located at the ventral midline (VM) of the spinal cord. Subsequently, most postcrossing commissural axons turn rostrally into the longitudinal plane and project alongside the FP [medial longitudinal commissural (MLC)] or grow into an intermediate region

\footnotetext{
Received Nov. 5, 2007; revised July 12, 2008; accepted July 15, 2008.

This work was supported by New York State Department of Health Spinal Cord Injury Research Board Grants C016886 and $\mathrm{C} 018615$ and National Institutes of Health Grants R01 NS038505 and 2R56NS038505 (Z.K.). S.L.R. was supported by Molecular Neuropathology Training Grant NS007098. We gratefully acknowledge S. Guthrie (King's College, London, UK) and J. Raper (University of Pennsylvania School of Medicine, Philadelphia, PA) for the truncated Robo and full-length Robo/control Del-Npn1 constructs, respectively. We thank T. Jessell and Susan Morton (Columbia University, New York, NY) for anti-Lhx2/9, and anti-Lhx1/5 (mAb 4F2), anti-Shh (5E1), anti-Nkx2.2 (74.5A5), and anti-SC1 (BEN) were obtained from the Developmental Studies Hybridoma Bank, developed under the auspices of the National Institute of Child Health and Human Development and maintained by the Department of Biological Sciences, University of lowa (lowa City, IA). We especially thank C. Krull (University of Michigan, Ann Arbor, MI) and C. Chesnutt (Sloan-Kettering Institute, New York, NY) for help troubleshooting our electroporation protocol. We also thank C. Mason (Columbia University, New York, NY), J. Raper, R. Imondi (Coastal Marine Biolabs, Ventura, CA), Cristina Aguirre-Chen, Ed Carlin, Arlene Bravo, and Angela Jevince for critical comments on this manuscript, $S$. Kadison and A. Dillon for insightful comments on this study, and M. Gulinello (Albert Einstein College of Medicine, Bronx, NY) for assistance with DeltaGraph software.

Correspondence should be addressed to Zaven Kaprielian, Department of Pathology and Dominick P. Purpura Department of Neuroscience, Albert Einstein College of Medicine, Kennedy Center, Room 624,1410 Pelham Parkway, South, Bronx, NY 10461. E-mail: kapriel@aecom.yu.edu.

DOI:10.1523/JNEUROSCI.1479-08.2008

Copyright $\odot 2008$ Society for Neuroscience $\quad 0270-6474 / 08 / 288698-11 \$ 15.00 / 0$
}

of the spinal cord [intermediate longitudinal commissural (ILC)] (Imondi and Kaprielian, 2001; Kadison and Kaprielian, 2004), but never recross the FP. We originally identified MLC and ILC axons through unilateral DiI labeling, which provides unobstructed views of postcrossing trajectories but cannot reproducibly mark MLC and ILC axons and reliably assess their behavior in response to perturbations. The bHLH (basic helixloop-helix) transcription factors, Atoh1 and Neurog1, define nonoverlapping progenitor populations that differentiate into genetically distinct dI 1 and $\mathrm{dI} 2$ commissural neurons, respectively (Gowan et al., 2001). Because Atoh1 and Neurog1 enhancers direct reporter gene expression to dI1 and dI2 neurons/axons in transgenic mice (Lumpkin et al., 2003; Nakada et al., 2004), they may provide useful tools for labeling subsets of contralateral commissural projections.

After crossing the VM, commissural axons lose their responsiveness to midline attractants and gain responsiveness to the midline-associated repellents, Slit1-3, in vitro. In mammals, the Slit-binding repulsive Robo receptors, Robol/2 (Long et al., 2004), are believed to be key components of the molecular mechanisms that switch the responsivity of these axons (Zou et al., 2000; Dickson and Gilestro, 2006). Accordingly, a third Robo, Robo3, represses the function of Robo1/2 on precrossing axons (Sabatier et al., 2004) and an interaction between the cytoplasmic domains of Robo1/2 and the attrac- 
tive Netrin-1 receptor, deleted in colorectal cancer (DCC), silences the attraction of postcrossing commissural axons to the FP (Stein and Tessier-Lavigne, 2001). These events presumably ensure that decussated commissural axons are selectively responsive to Slits, facilitating their expulsion from the VM and preventing their reentry into the FP. Consistent with this view, longitudinally projecting decussated commissural axons assume inappropriate positions relative to the FP in Slit or Robo mutant mice and recross the FP in the absence of Slits (Long et al., 2004). Nevertheless, the specific roles of Robo1/2 have not been delineated in vivo and some commissural axons elaborate appropriate contralateral projections in mice lacking Robo1, Robo2, or all three Slits (Long et al., 2004).

Here, we exploit unilateral in ovo electroporation in chick embryos to reliably mark commissural axons, including the dI 1 and dI2 subclasses, which elaborate ILC and MLC projections. We then use this labeling system to assess the consequences of manipulating Robo expression on these axons in vivo. In response to misexpressing cytoplasmic truncations of Robo1 and/or Robo2, which likely interfere with all Roboligand interactions, postcrossing commissural axons extend alongside the FP, but fail to project away from and do not reenter the FP. In contrast, misexpressing FL Robo2 prevents many commissural axons from crossing the VM. These findings support key in vivo roles for Robo receptors in presumably altering the responsiveness of postcrossing commissural axons and promoting their expulsion from the VM within the chick spinal cord.

\section{Materials and Methods}

In ovo electroporation. Fertilized White Leghorn eggs (Spafas) were incubated at $39^{\circ} \mathrm{C}$. Plasmid DNA diluted in a $0.15 \%$ trypan blue solution was unilaterally electroporated into stage $17 / 18$ or $18 / 19$ chick embryos (Hamburger and Hamilton, 1951) at the thoracic level of the spinal cord. Square-wave current (five pulses, $20 \mathrm{~V}, 50 \mathrm{~ms}$ ) was generated using a CUY-21 (Protech International) electroporator (Krull, 2004). The various constructs were electroporated at the following concentrations: enhancers $(1.5-2 \mu \mathrm{g} / \mu \mathrm{l})$, truncated Robos $(0.7 \mu \mathrm{g} / \mu \mathrm{l})$, full-length (FL) rRobo2/control del-Npn1 $(1.5 \mu \mathrm{g} / \mu \mathrm{l})$, and short-hairpin RNA (shRNA) $(2.5 \mu \mathrm{g} / \mu \mathrm{l})$. Open-book preparations were then generated from unfixed electroporated embryos as previously described (Imondi et al., 2000). Alternatively, the embryos were fixed, cryoprotected, and cryosectioned, or embedded in $8 \%$ sucrose $/ 3.5 \%$ agarose and vibratome-sectioned (40 $\mu \mathrm{m})$.

Electroporation constructs. The Atoh1tauGFP and Atoh1taumCherry constructs contain the $1.4 \mathrm{~kb}$ Atoh1 (previously Math1) enhancer (Lumpkin et al., 2003), the $\beta$-globin basal promoter (Yee and Rigby, 1993), and Tau sequences fused to EGFP (enhanced green fluorescent protein) (Clontech) or mCherry (Shaner et al., 2005) for axonal localization. The Neurog1tauGFP and Neurog1taumCherry constructs contain a $0.7 \mathrm{~kb}$ dorsal neural tube-specific Neurogl enhancer (TgN1-13) (Nakada et al., 2004). The CMV-Robo1 $\Delta-G F P$ and $C M V$ Robo $2 \Delta-G F P$ constructs contain sequences encoding truncated Robo green fluorescent protein (GFP) fusion proteins representing the Robo extracellular and transmembrane domains driven by a $C M V$ enhancer and a $\beta$-actin promoter (Hammond et al., 2005). The FL $\operatorname{rat}(r)$ Robo 2 construct is myc tagged and is driven by a $C M V$ enhancer and a $\beta$-actin $/ \beta$-globin promoter. The control del-Npn1 construct is driven by the same elements and directs the expression of a nonfunctional, myc-tagged protein consisting of the transmembrane and cytoplasmic domain of Npn1. We used the Ambion pSilencer 1.0-U6 vector that harbors a mouse U6 RNA polymerase III promoter to drive expression of short hairpin RNAs (Ambion). The production of the shRNA constructs was performed as previously described (Chesnutt and Niswander, 2004) and according to the Ambion protocol. The targeted sequences are as follows: 5'-AATGATGCTGGCAAG-
TACGTT-3' (RobolA43), 5' -AAGATGGGACTCCTCTAGATG-3' (Robo1B36), 5'-AATAGATCGCTAGCGTAGGCT-3' (RobolScram), 5' AACCCCTACTATTGAATGGTA-3' (Robo2A48), and 5'-AAGGAACTATCTTGGAGAAGC-3' (Robo2B56), and the oligonucleotides were synthesized by Gene Link.

Immunohistochemistry. Anti-Lhx2/9 was obtained from S. Morton and T. Jessell (Columbia University, New York, NY), and antiLhx1/5, anti-Shh (5E1), anti-Nkx2.2 (74.5A5), and anti-SC-1 (BEN) were obtained from the Developmental Studies Hybridoma Bank (University of Iowa, Iowa City, IA). Anti-GFP Alexa Fluor 488 conjugate, Alexa Fluor 488 goat anti-mouse, and Alexa Fluor 488 goat anti-rabbit were obtained from Invitrogen. Anti-myc (9E10) was obtained from Millipore Bioscience Research Reagents. Anti-cRobo1 and anti-cRobo2 polyclonal antibodies were commercially prepared (Genemed Biotechnologies) against peptides representing extracellular sequences within chick Robol (SNLAVTVDDSAEFKC; peptide 224) and Robo2 (INQVVLEEEAVDFRC; peptide 230). We confirmed the specificities of these new polyclonal antibodies by using immunodot assays (Ou et al., 1991) to show that anti-cRobol and anticRobo2 selectively bind their peptide immunogens (data not shown). We also performed preabsorption experiments by first incubating anti-cRobo 1 with peptide 224 or 230 and anti-cRobo 2 antiserum with peptide 230 or 224 ( $100 \mu \mathrm{g}$ of each peptide diluted in PBS) for $1 \mathrm{~h}$. Subsequently, we applied these mixtures to serial transverse cryosections of embryonic day 5 (E5) chick spinal cord and showed that preabsorption of anti-cRobol with peptide 224 , but not 230 , and anti-cRobo2 with peptide 230 , but not 224 , significantly attenuated antibody binding (see Fig. 3D,F). Anti-rat (r) Robol or anti-human (h) Robo2 polyclonal antibodies were obtained from R\&D Systems. In all cases, immunolabeling of sections was performed as previously described (Jevince et al., 2006) except that 3\% donkey and 5\% goat serum (Gemini Bio Products) was included in the blocking buffer (0.1\% Triton X-100 in PBS) used for the anti-rRobo/hRobo and cRobo antibodies, respectively, and primary antibody incubations were performed overnight at $4^{\circ} \mathrm{C}$. Appropriate Cy3-conjugated secondary antibodies (Jackson ImmunoResearch) were used to detect the binding of the various primary antibodies.

In situ hybridizaton. The cRobol/2 and cNetrin-1 cDNAs were obtained from E. Laufer (Columbia University, New York, NY) (Vargesson et al., 2001) and M. Tessier-Lavigne (Genentech, South San Francisco, CA) (Serafini et al., 1996), respectively, and each cDNA was linearized and transcribed as previously described (Dillon et al., 2005). Fluorescent in situ hybridization was performed by adapting a previously described nonradioactive protocol (Kaprielian et al., 1995) as follows: a $15 \mathrm{~min}$ incubation with $3 \%$ hydrogen peroxide was used to inactivate endogenous peroxidase activity, a 60 min incubation in $0.1 \%$ Triton X-100 was used in place of a proteinase $\mathrm{K}$ treatment to permeabilize the tissue, the cryosections were prehybridized/hybridized at $60^{\circ} \mathrm{C}$, anti-digoxigeninPOD (peroxidase), Fab fragments (Roche) were preadsorbed with E3.5/E4 chick embryo extracts, and probe hybridization was detected with the TSA Plus Cy3 Fluorescence kit (PerkinElmer; catalog \#NEL74400KT).

Photodocumentation. Reporter expression was visualized through epifluorescence optics (Nikon Eclipse TE300) using Cy2 or Cy3 filters (Chroma Technology). Colored images were captured and processed as previously described (Kadison and Kaprielian, 2004). Confocal images were acquired on a Fluoview 500 microscope (Olympus) using Cy2 and Cy3 filters. The images in Figures 1,D and $E$, and 4, and supplemental Figures 1 and 7 (available at www.jneurosci.org as supplemental material) represent confocal stacks.

Data analysis and imaging. We performed two separate analyses to quantify the axon pathfinding phenotype displayed by embryos coelectroporated with either Atoh1tauGFP or Neurog1tauGFP and $C M V$-Robo2FL-myc (Rb2). In one approach, we used epifluorescence microscopy and focused through the planes of the FP to count the total number of axons that crossed the VM and reached the contralateral margin of the FP in open-book preparations derived from these embryos. Subsequently, we compared these counts with those obtained from embryos electroporated with Atoh1tauGFP or 
NeurogltauGFP [wild type (WT)]. The mean number of axons reaching the contralateral margin of the FP was calculated in each of these groups (nine embryos per group). Two separate $t$ tests were used to compare the number of Atoh1 - and Neurog1-labeled axons in WT embryos and embryos misexpressing FL Robo2 using DeltaGraph software.

In a separate analysis, we objectively compared the relative number of GFP-positive axons projecting into the longitudinal plane on the ipsilateral side of the FP using confocal microscopy (Zeiss LSM 5 Duo V2: $488 \mathrm{~nm}$ line excitation; $20 \times$ objective, 0.8 numerical aperture) and quantitative digital image analysis (MetaMorph software; Molecular Devices). Each open-book preparation was rotated to position the FP at the top of the microscopic scan field $(2048 \times 2048$ pixels $)$, to view axons projecting into the longitudinal plane on the ipsilateral side. Then six line scans (at $0.22 \mu \mathrm{m} X Y$ pixel dimensions) were taken, each stepping through the full depth (with $z$-intervals of $0.77 \mu \mathrm{m}$ ) of the preparation, to generate either $Y Z$ or $X Z$ images as follows. Four of the six lines extended along the full $y$-axis, thereby obtaining $Y Z$ planes that captured, in cross section, those axons that had turned into the longitudinal plane. One of the six lines extended along the $x$-axis, thereby obtaining an $X Z$ plane that captured, in cross section, GFP-positive axons located proximal to transfected cell bodies to meter the relative number of electroporated cell bodies in each sample. In addition, one of the six lines generated an $X Z$ image taken for each open-book preparation in a region away from territory containing GFP-positive cells to obtain background levels of fluorescence. After correction for background and thresholding to omit low intensity pixels, an "object" analysis was performed on the positive 12-bit digital images using the integrated morphometry analysis module of MetaMorph to obtain an unbiased measure of the relative number of longitudinally projecting axons. Using pixel area $(\geq 6)$, shape factor $(0.19-1)$, and ellipse factor $(0-3.0)$ criteria, the number of "objects," representing axons captured in cross section, was determined for each image. These criteria were used to eliminate random noise or debris, as well as to avoid counting the rare transversely projecting axons that happened to overlap a given $Y Z$ line scan. Data analysis and statistical testing were performed using GraphPad InStat 3 software. The data were found to display a non-Gaussian distribution by the Kolmogorov-Smirnov test, and thus nonparametric statistical testing was performed.

\section{Results}

Murine Atoh1 and Neurog1 regulatory elements direct reporter gene expression to $\mathrm{dI} 1$ and $\mathrm{dI} 2$ commissural neurons/axons, respectively, in the chick spinal cord

To determine whether the murine Atoh1 and Neurog1 enhancers (Timmer et al., 2001; Lumpkin et al., 2003; Nakada et al., 2004) direct reporter gene expression to commissural neurons/axons in the avian spinal cord, we unilaterally electroporated Atoh1tauGFP and Neurog1tauGFP reporter plasmids into E2.5 (stage 18-19) embryos. In these embryos, Atoh1- and Neurog1-enhancer elements drive tauGFP expression to dorsal domains of the spinal cord $48 \mathrm{~h}$ after electroporation (Fig. $1 A, B)$. In contrast, unilateral electroporation of a $C M V-G F P$ construct that produces a membrane-targeted, myristylated form of GFP (Hammond et al., 2005) labeled a large number of cells broadly distributed along the dorsal-ventral (D-V) axis of the spinal cord (Fig. 1C). To confirm the identity of the GFP-labeled cells in the embryos electroporated with Atoh1tauGFP or Neurog1tauGFP, we labeled sections derived from these embryos with markers of dI1 or dI2 neurons (Gowan et al., 2001). Appropriately, anti-Lhx2/9 (dI1) labels a subset of GFP-expressing cell bodies in the chick spinal cord electroporated with Atoh1tauGFP (Fig. 1D). Similarly, antiLhx1/5 (dI2) labels a subset GFP-expressing cell bodies in chick embryos electroporated with Neurog1tauGFP (Fig. 1E). Conversely, Atoh1 GFP-labeled and Neurog1 GFP-labeled cells do not express Lhx1/5 and Lhx2/9, respectively (data not shown). In each of the electroporated embryos, GFP-labeled commissural axons were observed to cross the FP and enter the ventral funiculus on the contralateral side of the VM (Fig. $1 A-C)$. 

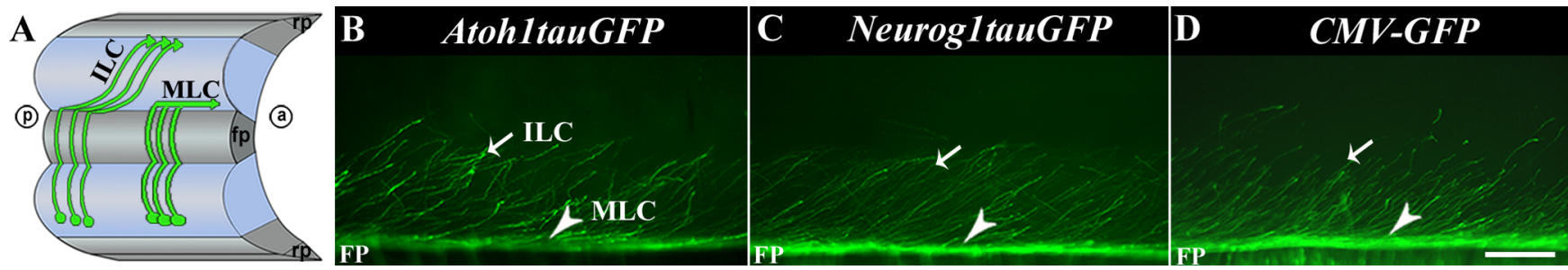

Figure 2. $\mathrm{d} 11$ and dI2 commissural axons elaborate ILC and MLC projections on the contralateral side of the FP. A, Schematic representing an open-book view of the spinal cord after the electroporation of GFP reporter constructs. In wild-type embryos, postcrossing MLC axons extend alongside the contralateral margin of the FP and ILC axons project a significant distance away from the FP. a, Anterior; $p$, posterior; $f p$, floor plate; rp, roof plate. $\boldsymbol{B}-\boldsymbol{D}$, Open-book preparations derived from E5 chick embryos unilaterally electroporated with Atoh 1tauGFP (B), Neurog 1 tauGFP ( $\boldsymbol{C}$, or CMV-GFP (D) reporter constructs. In each case, the labeled postcrossing axons elaborate both MLC (arrowheads) and ILC (arrows) projections. Scale bar, $100 \mu \mathrm{m}$.

Postcrossing dI 1 and dI 2 commissural axons elaborate ILC and MLC projections

To characterize the projection patterns of postcrossing axons emanating from dI1 and dI2 commissural neurons, we generated open-book preparations derived from chick embryos unilaterally electroporated with Atoh1tauGFP and Neurog1tauGFP, respectively. Both of these reporter constructs labeled rostrally projecting ILC and MLC axons (Fig. $2 A$, schematic; B, C) (Kadison and Kaprielian, 2004). Chick embryos electroporated with the CMV-GFP reporter construct display a similar set of contralateral projections (Fig. 2D); however, a significant subset of the labeled ILC axons projects in the caudal direction (data not shown). Thus, although this marking system can be used to distinguish between dI1 and dI2 commissural neurons and facilitates the visualization of their axons, each population projects along both ILC and MLC trajectories on the contralateral side of the FP.

Robol and Robo2 are expressed on postcrossing segments of commissural axons in the chick spinal cord

In the mouse spinal cord, Robol/2 are preferentially expressed on postcrossing segments of commissural axons (Long et al., 2004). To determine whether Robos are expressed in a similar manner in the avian spinal cord, we first used in situ hybridization to analyze the distribution of robol and robo 2 mRNA in transverse sections derived from stage 23-24 (E4) chick embryos when commissural axons have crossed the FP (Stoeckli and Landmesser, 1995). Dorsal domains of robol and robo 2 mRNA expression overlap with the positions occupied by a variety of interneuron cell bodies (Fig. $3 A, B$, arrow). We also labeled transverse cryosections derived from stage 26-27 (E5) chick embryos with anti-cRobol and anti-cRobo2 polyclonal antibodies that recognize unique sequences within the extracellular domains of chick Robol and Robo2 (see Materials and Methods). Consistent with findings in the embryonic mouse spinal cord, Robol/2 proteins are enriched in the decussated commissural axon-containing marginal zone (Fig. $3 C, E$, arrow).

\section{Subsets of dI1 and dI 2 commissural neurons express robo 1 and robo 2 mRNA}

To determine whether $\mathrm{dI} 1$ and $\mathrm{dI} 2$ commissural neurons express Robol/2, we performed fluorescence in situ hybridization for robo1 or robo $2 \mathrm{mRNA}$ on transverse cryosections derived from $\mathrm{E} 4$ chick embryos electroporated with the Atoh1-tauGFP or Neurog1-tauGFP reporter constructs. Confocal microscopy revealed that at least subsets of Atoh1- and Neurog1-GFP- expressing cells contain robo1 and robo2 mRNA (Fig. 4A-D). We confirmed these observations by double-labeling transverse cryosections derived from $\mathrm{E} 4$ chick embryos with in situ hybridization probes for robo1 or robo2, as well as anti-Lhx2/9 or anti-Lhx1/5 (supplemental Fig. $1 A-D$, available at www.jneurosci.org as supplemental material).

\section{Postcrossing commissural axons that express a truncated} form of Robo1 or Robo2 project alongside the FP, but fail to project away from the VM along ILC trajectories

Loss-of-function studies performed in the mouse spinal cord have shown that many commissural axons elaborate appropriate contralateral projections in the absence of Robo1 or Robo2 (Long et al., 2004). Therefore, we took an alternative approach to determine the function(s) of Robo proteins in the chick spinal cord by assessing the consequences of disabling all Robo signaling in commissural axons. Cytoplasmic domain-lacking forms of Robos have previously been used to reduce or abrogate Robo function, presumably in a dominant-negative manner by disrupting Robo-ligand interactions, in a variety of in vitro and in vivo systems (Stein and Tessier-Lavigne, 2001; Whitford et al., 2002; Hammond et al., 2005; Jia et al., 2005). Accordingly, we asked whether unilaterally electroporating constructs encoding truncated Robo1 or Robo2 into stage 17-18 (E2.5) chick embryos would perturb commissural axon pathfinding. Initially, we used the Atoh1 enhancer in an attempt to drive truncated forms of Robol or Robo2 to dI1 neurons/axons; however, this resulted in essentially undetectable levels of ectopic expression. Therefore, we electroporated chick embryos with $C M V$-Robo1 $\Delta-G F P$ and $C M V$-Robo2 $\Delta-$ GFP constructs that encode the extracellular and transmembrane domains of human Robo1 or Robo2, respectively. Labeling of sections derived from the electroporated embryos with anti-rat Robol and anti-human Robo 2 confirmed the unilateral misexpression of truncated Robo1/Robo2 proteins (supplemental Fig. 2, available at www.jneurosci.org as supplemental material). We then generated open-book preparations to analyze the projections of the labeled commissural axons on the contralateral side of the FP. In contrast to chick embryos that had been electroporated with CMV-GFP (control) (Fig. 5A-C), none of the labeled axons extended away from the ventral midline in embryos electroporated with $C M V$-Robo1 $\Delta$-GFP (Fig. 5D-F; supplemental Fig. 3B, available at www.jneurosci.org as supplemental material), $C M V$ Robo2 $\Delta$-GFP (supplemental Fig. 3C, available at www.jneurosci.org as supplemental material), or both $C M V$-Robo1 $\Delta-G F P$ and $C M V$-Robo $2 \Delta-G F P$ (data not shown). Although these axons crossed the VM and turned into the longitudinal plane by 
E4 (36 h post electroporation; observed in 10 of 10 embryos) (Fig. 5D), they projected alongside the contralateral margin of the FP elaborating only MLC, rather than both MLC and ILC, projections between E5 ( $48 \mathrm{~h}$; observed in 11 of 11 embryos) (Fig. 5E) and E6 (72 h; observed in 6 of 6 embryos) (Fig. 5F). To exclude the possibility that misexpressing a truncated form of Robo affects the normal properties of the FP, and/or other midline-associated cells, we analyzed the expression pattern of Shh, a key FP-associated morphogen (Charron et al., 2003), $\mathrm{Nkx} 2.2$, a homeodomain-containing protein that is expressed in cells located immediately adjacent to the FP (Briscoe et al., 1999), BEN, a cell surface protein that is expressed by FP cells (Weiner et al., 2004), and Netrin-1, a FP-derived chemoattractant for commissural axons (Serafini et al., 1996), in embryos electroporated with $C M V$-Robo1 $\Delta-G F P$. These labeling studies revealed a wild type-like distribution of $\mathrm{Shh}, \mathrm{BEN}$, and netrin-1, suggesting that misexpression of a truncated form of Robo-1 does not indirectly perturb commissural axon guidance by altering the normal properties of the FP or immediately adjacent cells (supplemental Fig. $4 A-D$, available at www.jneurosci.org as supplemental material). To assess the consequences of misexpressing truncated Robo receptors on the pathfinding of $\mathrm{dI} 1$ and $\mathrm{dI} 2$ axons, we unilaterally coelectroporated E2.5 chick embryos with Atoh1taumCherry or NeurogltaumCherry and CMV-Robo1 $\Delta-G F P$ or CMV-GFP. Consistent with our findings described above, the labeled postcrossing dI 1 and dI 2 commissural axons failed to project away from the FP (Fig. 6C,F). Notably, these manipulations did not differentially affect the guidance of dI1 and dI2 axons. Similar results were observed when $C M V-R o b o 2 \Delta-G F P$ was coelectroporated with $\mathrm{dI} 1$ and $\mathrm{dI} 2$ reporter constructs (data not shown). Thus, although axons bearing truncated Robo receptors cross the floor plate and elaborate MLC projections, they do not project away from the VM along ILC trajectories.

\section{Unilateral electroporation of truncated Robol prevents} postcrossing commissural axons on either side of the spinal cord from projecting away from the FP

Given that misexpressed cytoplasmic truncations of Robo likely bind all available Robo ligands (i.e., midline-derived Slits), even those postcrossing axons, which do not express truncated Robo should fail to project away from the FP. To test this prediction, we asked whether the pathfinding of axons arising from the contralateral side of the spinal cord (Fig. $7 A$, schematic; red axons) is perturbed in response to the unilateral misexpression of truncated Robo receptors by electroporating NeurogltaumCherry (Fig. 7A, schematic; red axons) into one side of an E2.5 chick embryo and, $2 \mathrm{~h}$ later, electroporating the opposite side with $C M V$-Robo1 $\Delta-G F P$ (Fig. 7A, schematic; green axons). As expected, in open-book preparations derived from these embryos $48 \mathrm{~h}$ after the final electroporation, GFP-labeled axons emanating from the side of the spinal cord electroporated with $C M V$ Robo $1 \Delta-G F P$ failed to project away from the FP (Fig. $7 A$, $B$, schematic, dashed line; green axon). Consistent with the unilateral misexpression of truncated Robol perturbing commissural axon pathfinding in a bilateral manner, postcrossing segments of mCherry-labeled dI2 axons arising from the opposite side of the spinal cord also failed to extend away from the FP (Fig. 7A, $B$, schematic, dashed line; red axon; observed in 11 of 11 embryos). These robust phenotypes contrast our finding that, in embryos coelectroporated with CMV-GFP and Neurog1taumCherry,
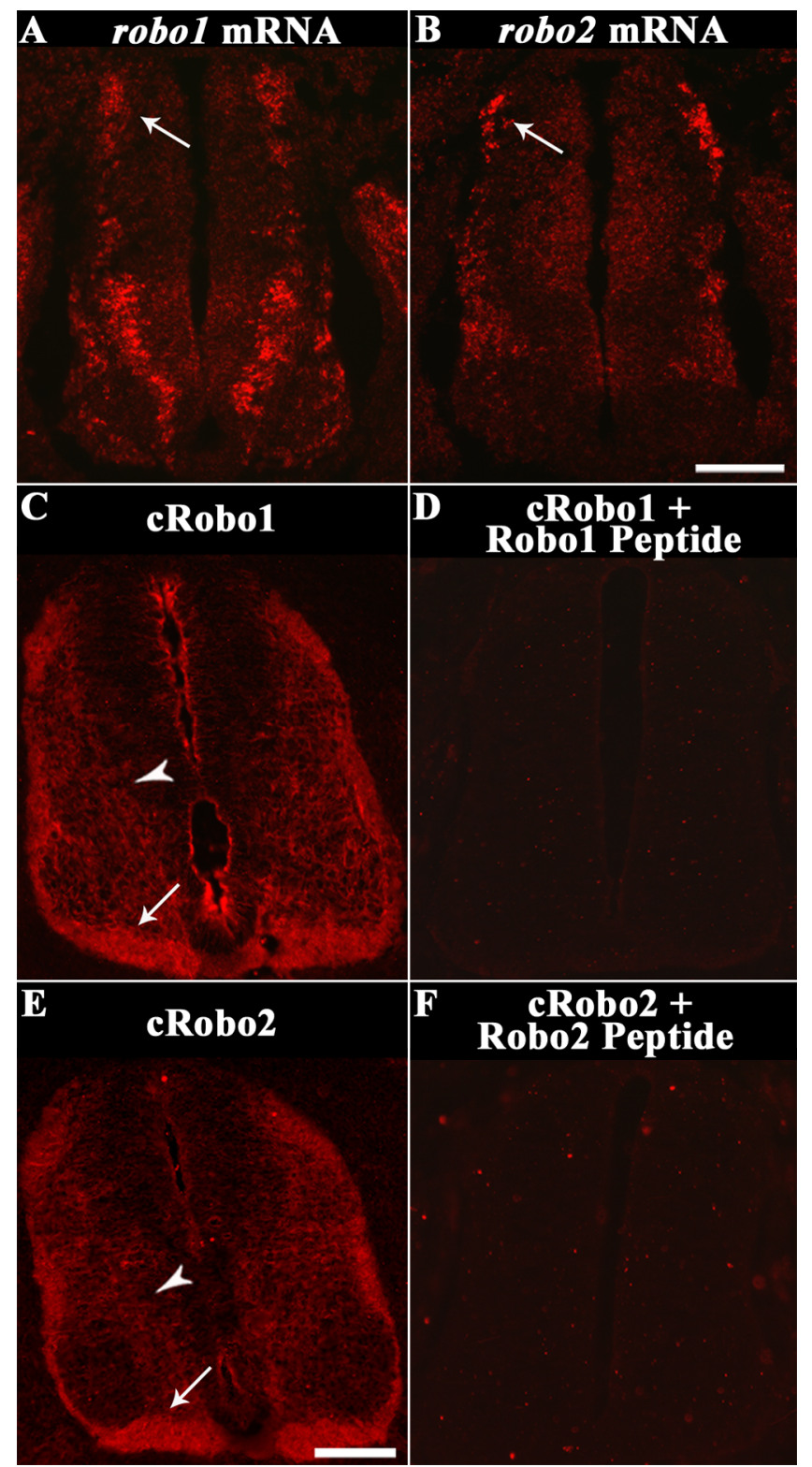

Figure 3. Robo1 and Robo2 are preferentially expressed on postcrossing segments of commissural axons that arise from dorsal interneurons in the chick spinal cord. $\boldsymbol{A}, \boldsymbol{B}$, Fluorescent in situ hybridization performed on transverse cryosections derived from E4 chick spinal cords with chick robo1 $(\boldsymbol{A})$ and $\operatorname{robo2}_{(\boldsymbol{B})}$ riboprobes reveals domains of mRNA expression that coincide with the positions of dorsal interneurons (arrows). C, E, Both anti-cRobo1 and anti-cRobo2 strongly label the decussated commissural axon-rich marginal zone (arrows), and weakly label precrossing commissural axons (arrowheads) in transverse cryosections derived from E5 chick embryos. D, F, Preincubating anti-cRobo1 and anti-cRobo2 with their peptide immunogens for $1 \mathrm{~h}$ significantly diminished the binding of these antibodies to E5 chick spinal cord sections. Conversely, anti-cRobo1 and anti-cRobo2 staining is not attenuated by preabsorption with the Robo2 and Robo1 peptide immunogens, respectively (data not shown). Scale bars, $100 \mu \mathrm{m}$.

postcrossing commissural axons extend along both MLC and ILC trajectories (Fig. 7C). Consistent with our prediction, these observations suggest that misexpression of a truncated form of Robo prevents even those axons that express only endogenous Robos from elaborating ILC projections on the contralateral side of the FP. Presumably, this is because the truncated Robos sequester FP-associated Robo ligands away from the endogenous FL receptors. 

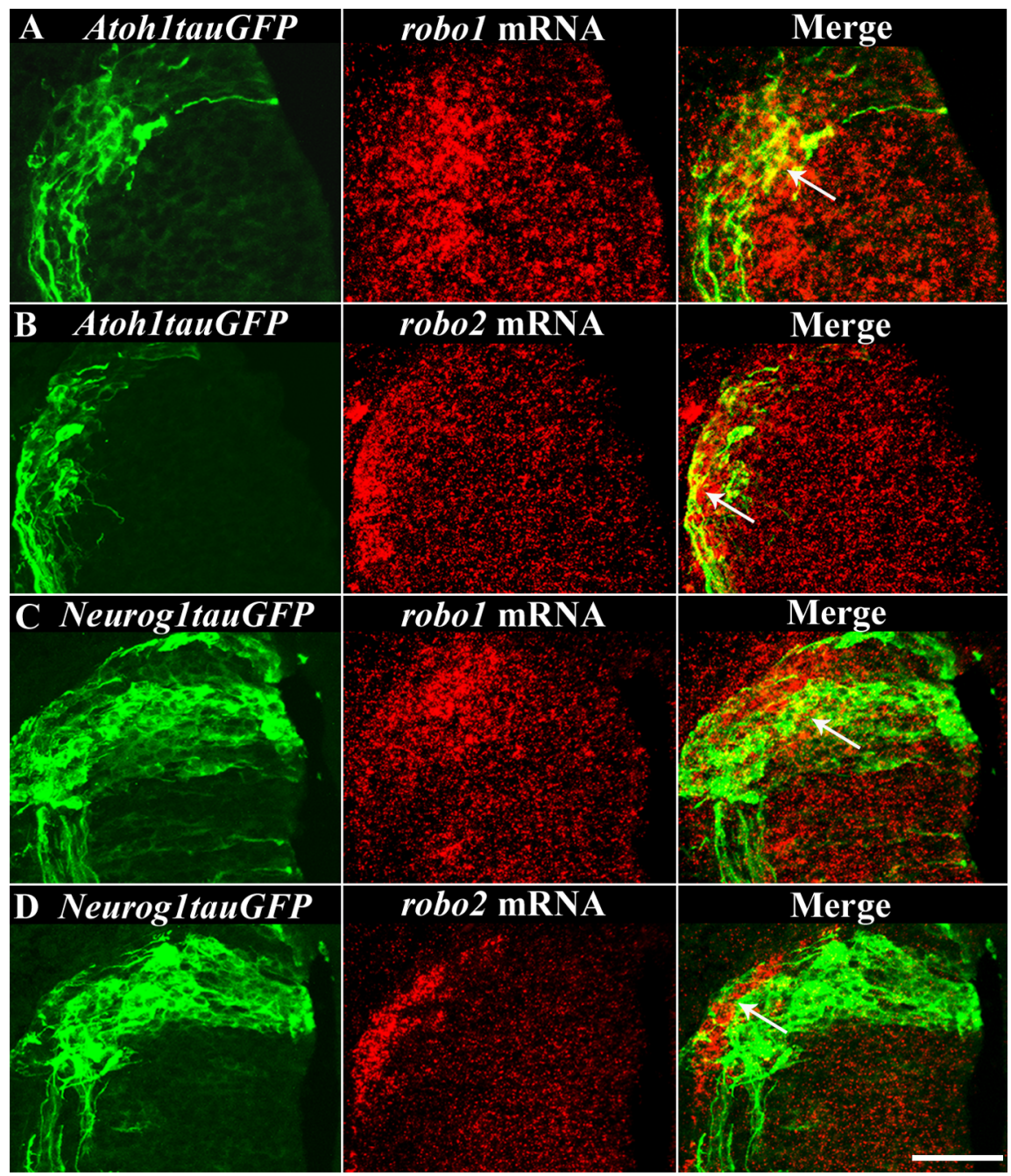

Figure 4. Subsets of $\mathrm{d} 11$ and $\mathrm{dl} 2$ commissural neurons express robo 1 and robo2 mRNA. A-D, Transverse cryosections derived from E4 chick embryos electroporated with either Atoh 1tauGFP or Neurog 1 tauGFP were subjected to fluorescent in situ hybridization to detect robo 1 and robo2 mRNA followed by anti-GFP labeling to identify d 1 and d 2 commissural neurons. In each row, the first panel represents GFP expression within the dorsal quadrant of the left side of the spinal cord (Fig. $1 A, B$ ), the second panel represents Cy3-labeled robo 1 or robo2 mRNA expression, and the third panel is the merge of the first two panels. dl1 $(A, B)$ and d12 $(\boldsymbol{C}, \boldsymbol{D})$ dorsal commissural neurons express robo1 $(\boldsymbol{A}, \boldsymbol{C})$ and robo2 $(\boldsymbol{B}, \boldsymbol{D})$ mRNA (see arrows in third panel). Scale bar: (in $\boldsymbol{D}) \boldsymbol{A}-\boldsymbol{D}$, $50 \mu \mathrm{m}$.

\section{Misexpressing FL Robo2 prevents commissural axons from crossing the FP}

The segregation of Robos to postcrossing segments of commissural axons presumably ensures that, as has been observed in vitro (Zou et al., 2000), these axons gain responsiveness to midline-associated Slits only after they cross the FP. To test this hypothesis in vivo, we unilaterally electroporated $C M V$ Robo2FL-myc into E2-E2.5 chick embryos and assessed the consequences of precociously expressing Robo 2 on commissural axons. In contrast to embryos electroporated with a control construct (del-Npn1) (see Materials and Methods), in which most anti-myc-labeled axons crossed the FP (Fig. $8 \mathrm{~A}$, inset), misexpression of FL Robo 2 prevented nearly all commissural axons from crossing the VM (Fig. $8 B$, inset). Antimyc labeling of open-book preparations derived from these electroporated embryos confirmed that FL Robo2 is expressed on precrossing commissural axons (supplemental Fig. 5, available at www.jneurosci.org as supplemental material). Misex- pression of FL Robo2 similarly prevented a significant number of $\mathrm{dI} 1$ and dI2 axons from crossing the VM in embryos coelectroporated with Atoh1tauGFP or Neurog1tauGFP and CMVRobo2FL-myc (Fig. 8D, inset; E) (see Materials and Methods), compared with embryos electroporated with Atoh1tauGFP (Fig. 8E) or Neurog1tauGFP (Fig. $8 C$, inset; $E$ ). Consistent with their presumed lack of FL Robo2, essentially all of the GFP-expressing axons that crossed the VM were not labeled by antimyc (supplemental Fig. 6, available at www.jneurosci.org as supplemental material).

Paralleling a reduction in the number of FP-crossing axons in embryos electroporated with $C M V$-Robo2FL-myc alone or coelectroporated with $C M V$ Robo2FL-myc and Neurog1tauGFP, we expected to observe an increase in the number of longitudinally projecting axons on the ipsilateral side of the FP in the latter group of embryos, compared with those electroporated with CMV-DelNpn1-myc or Neurog1tauGFP. It is important to note in this regard, however, that the CMV-Del-Npn1-myc and CMVRobo2FL-myc (supplemental Fig. 5, available at www.jneurosci.org as supplemental material) constructs target a large number of cells distributed along the $\mathrm{D}-\mathrm{V}$ axis, and that this obscures axons on the ipsilateral side of the FP. To overcome this limitation, we analyzed GFP-labeled axons emanating selectively from dorsally located dI 2 cell bodies in open-book preparations derived from embryos coelectroporated with $C M V$ Robo2FL-myc and Neurog1tauGFP. Consistent with a decrease in the number of axons crossing the FP in these embryos (Fig. $8 E$, graph), there appeared to be an increase in the number of GFPlabeled, longitudinally oriented axons on the ipsilateral side of the spinal cord (Fig. 9B, arrow), compared with the number of these axons in embryos electroporated with only Neurog1tauGFP (Fig. 9A, arrow). In a subset of the open-book preparations derived from embryos coelectroporated with Neurog1tauGFP and CMV-Robo2FL-myc, we used anti-BEN to demarcate the FP boundaries to confirm that the GFPlabeled axons, which inappropriately remained on the ipsilateral side of the spinal cord, do not enter the FP (supplemental Fig. 7, available at www.jneurosci.org as supplemental material).

To determine the number of axons projecting into the longitudinal plane on the ipsilateral side of the spinal cord in a given electroporated embryo, we performed quantitative image analysis of optical planes captured through the use of confocal fluorescence microscopy. As detailed in Materials and Methods, each open-book preparation was rotated to position the FP at the top of the microscopic scan field to view axons 
projecting into the longitudinal plane on the ipsilateral side of the spinal cord. Four vertical line scans were taken through the depth of each preparation to obtain $Y Z$ planes that captured, in cross section, those axons that had turned into the longitudinal plane. The raw data from these scans revealed a clear visual difference between the number of axons turning into the longitudinal plane in Neurog1tauGFP (Fig. 9A') compared with Neurog1tauGFP-CMVRobo2FLmyc (Fig. 9 $B^{\prime}$ ) electroporated embryos, and this was corroborated by statistical analyses of the number of objects classified as longitudinally projecting axons in each group (Table 1). To determine whether this difference could have been the result of an increased rate of transfection in the Neurog1tauGFP-CMVRobo2FLmyc electroporated embryos, a full-depth horizontal line scan was also taken from each preparation proximal to the positions of the transfected cell bodies (see Materials and Methods). Subsequent analyses of these data revealed no significant difference in the transfection efficiency between the two groups (Table 1). We also calculated the ratio of the number of longitudinally projecting axons (determined by the number of objects in each vertical line scan) to the total number of transversely projecting, labeled axons (determined from the horizontal line scans) in each preparation. The ratios of the median values were found to be highly significant and showed about a twofold difference between the Neurog1 (WT) and Neurog1/FL Robo2 (Rb2) embryos (Table 1). These findings are consistent with the approximately twofold decrease in the number of axons that cross the floor plate in embryos coelectroporated with Neurog1tauGFP and CMV-Robo2FL-myc (Fig. 8 E). Together, these observations suggest that axons misexpressing Robo2FL prematurely gain responsiveness to a Slit gradient emanating from the FP.

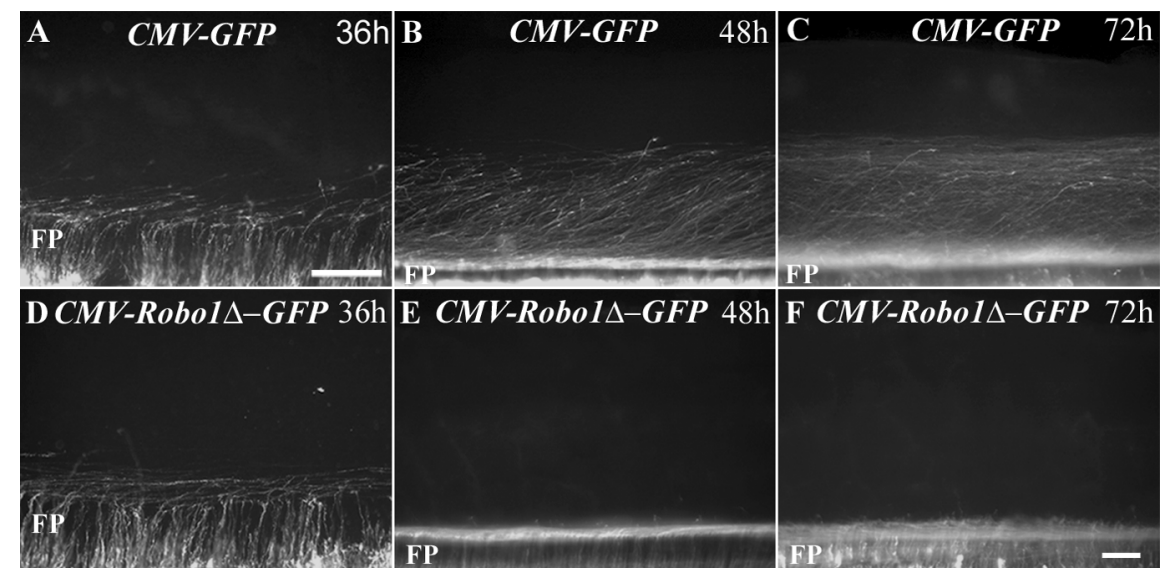

Figure 5. Postcrossing commissural axons expressing a truncated, cytoplasmic domain-lacking form of Robo1 fail to project away from the FP along ILC trajectories in the embryonic chick spinal cord. CMV-GFP (control) and CMV-Robo1 GFP constructs were separately and unilaterally electroporated into stage E2.5 chick embryos, and open-book preparations were derived from these embryos 36,48 , or $72 \mathrm{~h}$ after electroporation. $\boldsymbol{A}-\boldsymbol{C}$, In embryos electroporated with the CMV-GFP reporter construct, a significant number of commissural axons have crossed the floor plate and begun to extend away from the VM at E4, $36 \mathrm{~h}$ after electroporation $(\boldsymbol{A})$. Between E5 $(48 \mathrm{~h})(\boldsymbol{B})$ and $E 6(72 \mathrm{~h})(\boldsymbol{C})$, the postcrossing segments of these axons elaborate ILC and MLC projections. D-F, In CMV-Robo1 $\Delta$-GFP-treated embryos, GFP-labeled axons successfully cross and turn into the longitudinal plane alongside the FP at E4, $36 \mathrm{~h}$ after electroporation (D) (observed in 10 of 10 embryos). Although these axons continue to extend adjacent to the contralateral margin of the FP along MLC trajectories, they do not project away from the VM between E5 (48 h) (E) (observed in 11 of 11 embryos) and E6 (72 h) $(\boldsymbol{F})$ (observed in 6 of 6 embryos). Scale bars, $100 \mu \mathrm{m}$.
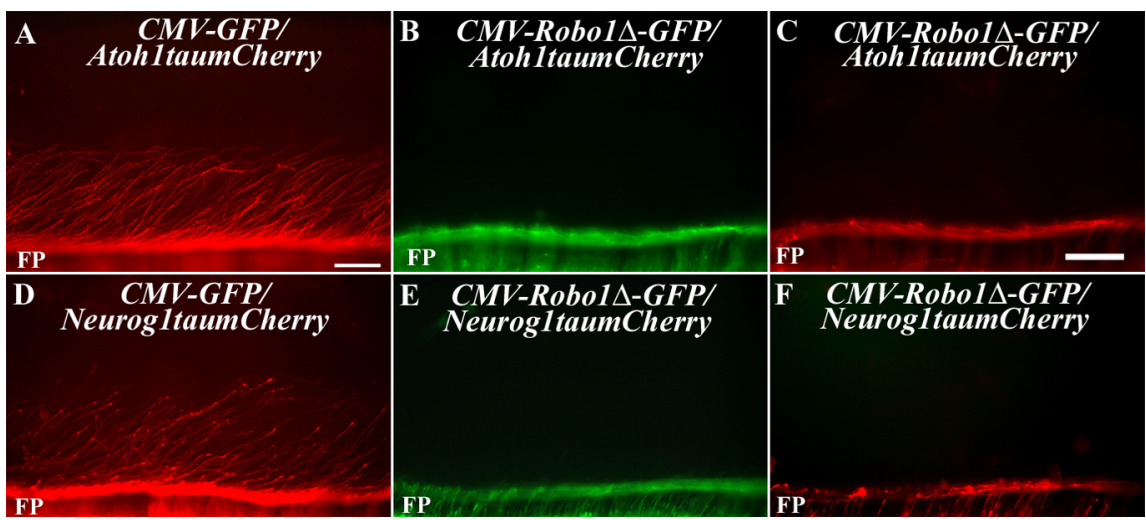

Figure 6. Postcrossing $\mathrm{dl} 1$ and $\mathrm{dI} 2$ commissural axons fail to project away from the FP in chick embryos unilaterally electroporated with a truncated form of Rob01. A, D, Open-book preparations derived from $\mathrm{E} 5$ chick embryos that had been coelectroporated with CMV-GFP and Atoh1taumCherry $(\boldsymbol{A})$ or Neurog1taumCherry $(\boldsymbol{D})$ at E2.5 contain labeled, postcrossing dl1 and dI2 axons, which have projected away from the FP along ILC and MLC trajectories. In an E5 chick embryo that had been coelectroporated with CMV-Robo1D-GFP and Atoh1taum Cherry (B, C) (8 of 8 embryos) or Neurog1taumCherry $(\boldsymbol{E}, \boldsymbol{F})$ ( 5 of 5 embryos) at E2.5, both GFP- $(\boldsymbol{B}, \boldsymbol{E})$ and $\mathrm{mCherry}$-labeled $(\boldsymbol{C}, \boldsymbol{F})$, postcrossing axons exclusively project adjacent to the contralateral margin of the FP along MLC trajectories. Scale bars, $100 \mu \mathrm{m}$.

\section{Discussion}

In the vertebrate spinal cord, most postcrossing commissural axons adopt MLC or ILC trajectories, projecting alongside or a significant distance away from the FP, respectively (Imondi and Kaprielian, 2001; Kadison and Kaprielian, 2004). The upregulation of Robo1/2 and the lack of Robo3 repression, as well as the loss of responsiveness to FP-associated chemoattractants, are believed to ensure that postcrossing commissural axons selectively gain responsive to repulsive midlineassociated Slit proteins (Dickson and Gilestro, 2006). Subsequently, Robo1/2-mediated repulsion presumably prevents all decussated commissural axons from reentering the FP and drives ILC axons away from the VM. However, these particular roles for Robo1/2 have not been established in vivo, and some commissural axons appear capable of adopting appropriately shaped contralateral trajectories even in the absence of repulsive Robo-Slit interactions (Long et al., 2004). Here, we exploit unilateral in ovo electroporation and use a variety of reporter constructs, including those that mark genetically distinct $\mathrm{dI} 1$ and $\mathrm{dI} 2$ axons, to reproducibly label MLC and ILC axons in the embryonic chick spinal cord and to reliably assess the consequences of manipulating Robo expression on their pathfinding. Disabling Robo signaling by misexpressing truncated Robo1/2, or misexpressing FL Robo2, on commissural axons leads to pathfinding defects that support 

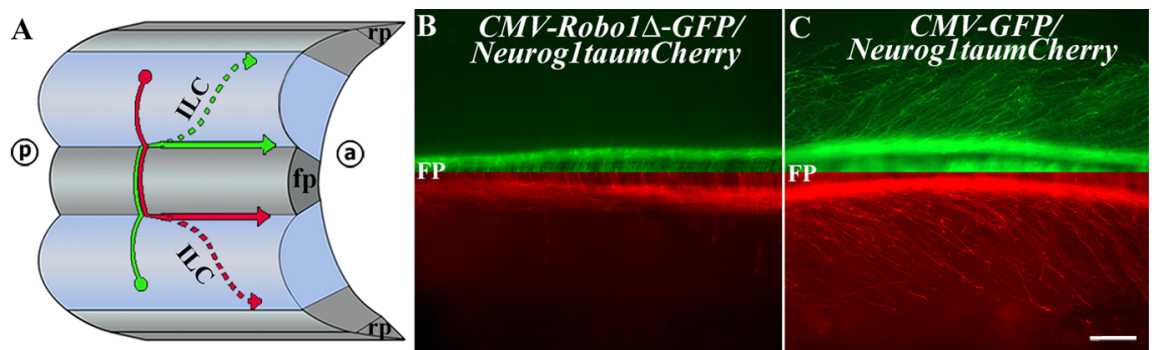

Figure 7. Postcrossing commissural axons on either side of the spinal cord fail to project away from the FP after unilateral electroporation of a truncated form of Rob01. A, Schematic depicting an open-book view of the spinal cord after the coelectroporation strategy described below. The dashed lines represent wild-type ILC projections, and the solid lines represent the trajectories that these axons adopt after unilateral electroporation of truncated Robo1. a, Anterior; $p$, posterior; fp, floor plate; $r p$, roof plate. $\boldsymbol{A}, \boldsymbol{B}$, Neurog1taum Cherry (red axons in schematic) was unilaterally electroporated into E2.5 chick embryos, and $\sim 2 \mathrm{~h}$ later the opposite side of the spinal cord was electroporated with CMV-Robo1 $\Delta$-GFP (green axons in schematic). At E5, postcrossing GFP-labeled axons misexpressing a truncated form of Robo1 do not project away from the $\mathrm{FP}(\boldsymbol{B})$ and postcrossing $\mathrm{dl} 2 \mathrm{mCherry}$-labeled axons emanating from cell bodies on the opposite side of the spinal cord also fail to project away from the FP (observed in 11 of 11 embryos). C, Open-book preparations derived from embryos coelectroporated with CMV-GFP and Neurog1taumCherry. Postcrossing axons on either side of the spinal cord elaborate wild-type MLC and ILC projections. Scale bar, $100 \mu \mathrm{m}$.
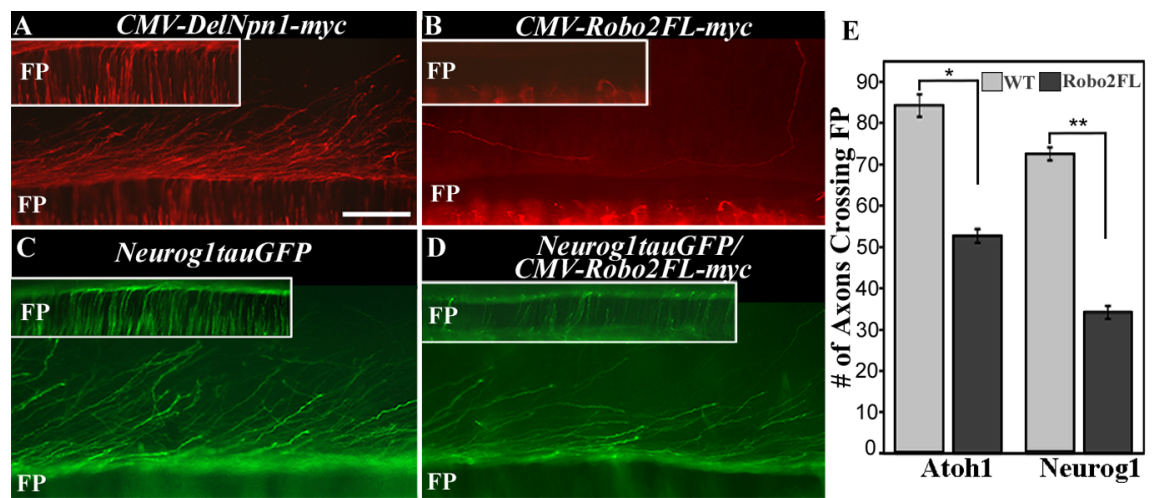

Figure 8. Commissural axons fail to cross the VM after unilateral electroporation of FL Robo2. $\boldsymbol{A}, \boldsymbol{C}$, Open-book preparations derived from E4-E4.5 chick embryos electroporated with a control CMV-Del-Npn1-myc plasmid $(\boldsymbol{A})$ (8 of 8 embryos) or the Neurog1tauGFP construct (C) ( 9 of 9 embryos) at E2.5 display wild-type ILC and MLC axons. $\boldsymbol{B}$, In embryos electroporated with CMV-Robo2FL-myc, essentially all myc-labeled axons fail to cross the FP ( 6 of 6 embryos). The two axons that do cross the FP follow aberrant trajectories on the contralateral side of the midline and this is indicative of the pathfinding behavior displayed by these rare axons. D, After the coelectroporation of Neurog1tauGFP and CMV-Robo2FL-myc, fewer labeled axons crossed the FP (D, inset) ( 9 of 9 embryos) compared with embryos electroporated with only Neurog1tauGFP(C, inset). $\boldsymbol{E}$, Bar graph quantifying the number of axons that cross the FP in embryos electroporated with Neurog1tauGFP or Atoh1tauGFP or coelectroporated with Neurog1tauGFP or Atoh1tauGFP and CMV-Robo2FL-myc. Approximately twofold fewer axons cross the FP in the embryos coelectroporated with Neurog1tauGFP or Atoh1tauGFP and CMV-Robo2FL-myc. Error bars indicate SEM. ${ }^{*} p<0.0001 ;{ }^{* *} p<0.0001$. Scale bar, $100 \mu \mathrm{m}$.

key in vivo roles for Robo receptors in presumably altering the responsiveness of decussated commissural axons and facilitating their expulsion from the VM.

\section{A genetic labeling strategy for visualizing postcrossing vertebrate commissural axons}

A key feature of the axonal labeling approach we use here is that, like DiI labeling, unilateral electroporation of commissural neurons provides unobstructed views of their postcrossing axons (Brittis et al., 2002). In contrast to DiI labeling, however, in ovo electroporation of specific reporter constructs ensures that the same subsets of contralateral commissural projections are reproducibly marked in each embryo. We show that murine Atoh 1 and Neurog 1 enhancers target dI 1 and dI2 neurons/axons, respectively, in chick embryos (Timmer et al., 2001). Although this labeling system distinguishes the dI1 and dI2 populations, and allows for the visualization of their axons, Atoh1, Neurog1, as well as $\mathrm{CMV}$ enhancers drive reporter protein expression to both MLC and ILC axons. Despite our observation that the Atoh1 and Neurog1 enhancer constructs do not label distinct subsets of postcrossing axons, these tools establish a genetic labeling system for reliably assessing the consequences of manipulating receptor expression on commissural axons.

\section{Assessing the role(s) of Robos in commissural axon guidance by manipulating receptor expression in vivo}

In mice lacking Robol or all three Slits, some commissural axons temporarily stall within the FP. In addition, decussated commissural axons inappropriately recross the VM in Slit triple knock-out embryos, and, in Robo1 or Robo2 null mice, longitudinally projecting, postcrossing commissural axons assume aberrant positions that are consistent with the more lateral location of Robo2- compared with Robo-1-expressing axons in wild-type embryos (Long et al., 2004). However, a soluble form of the extracellular domain (ECD) of Robo2 introduced into chick embryos perturbs the pathfinding of precrossing commissural axons. This particular observation raises the possibility that the Robo2 ECD may not simply interfere with ligand binding but might also bind other cell surface molecules and activate pathways required for guiding commissural axons to the FP (Mambetisaeva et al., 2005).

Given the range of pathfinding defects reported in these various in vivo systems, we used a misexpression strategy to explicitly assess the role(s) of Robos/Slits in the guidance of MLC and ILC axons. We show here that unilaterally misexpressing cytoplasmic truncations of Robo1/2 in the embryonic chick spinal cord prevents postcrossing commissural axons from projecting away from the FP along ILC trajectories, but does not disrupt the pathfinding of MLC axons. However, precocious expression of FL Robo2 prevents many commissural axons from crossing the FP. Together with our observation that endogenous Robol/2 proteins are preferentially expressed on postcrossing segments of chick commissural axons, these findings provide strong in vivo evidence supporting major roles for Robos in presumably altering the responsiveness of postcrossing axons to midline repellents and expelling these axons from the VM.

As a consequence of unilaterally misexpressing myc-tagged FL Robo2 via a CMV promoter within the embryonic chick 
spinal cord, essentially all myc-labeled axons failed to cross the FP. This robust phenotype is consistent with FP contact and the upregulation of Robol/2 altering the responsiveness of commissural axons to midline repellents (Zou et al., 2000) and suggests that misexpression of FL Robo2 overcomes the positive actions of Robo3, which normally facilitates midline crossing (Sabatier et al., 2004). Although we have not directly shown that axons misexpressing FL Robo2 gain responsiveness to repulsive midlineassociated Slits, our findings indicate that the precocious expression of a single repulsive Robo receptor on commissural axons is sufficient to prevent these axons from entering the FP. Moreover, many of these misguided axons prematurely turn into the longitudinal plane on the ipsilateral side of the spinal cord. Notably, a significant number of dI1/dI2 axons fail to cross the FP in embryos coelectroporated with the FL Robo2 and Atoh1 or Neurog1 reporter constructs. Appropriately, the subset of dI2 axons that is capable of crossing the FP in these experiments does not ectopically express FL Robo2.

Why does the unilateral misexpression of truncated Robos disrupt the pathfinding of postcrossing commissural axons on both sides of the FP? We initially presumed that, when expressed at high levels on commissural axons, truncated Robos would operate in a cellautonomous manner by dominating the response of the growth cone and overshadowing the function of endogenous Robos. Accordingly, the postcrossing segments of these axons would be incapable of responding to midline Slits, and as we have shown, they are not expelled from the FP and adopt MLC trajectories. However, decussated commissural axons that exclusively express endogenous Robos also fail to be expelled from the VM when truncated Robos are selectively expressed on axons arising from the opposite side of the spinal cord. Therefore, it seems likely that unilaterally misexpressed truncated Robos block the expulsion of decussated commissural axons on both sides of the spinal cord via a cell nonautonomous mechanism by presumably sequestering all midline-derived Slits. In vitro studies performed with Xenopus spinal cord neurons have established that a Slit-dependent interaction between the cytoplasmic domains of Robos and the Netrin receptor DCC "silences" the attraction of these axons to the FP, thereby facilitating their exit from the VM (Stein and Tessier-Lavigne, 2001). The cell nonautonomous model for the action of truncated Robos that we favor is consistent with a Slit-dependent, Robol/2-mediated silencing mechanism normally facilitating the expulsion of decussated commissural axons from the VM in the chick spinal cord.
Which guidance systems ensure that decussated commissural axons never reenter the FP?

We were surprised to find that decussated commissural axons did not reenter the FP after misexpression of truncated Robo $1 / 2$ in the chick spinal cord given that this manipulation likely removes all available midline Slits (see above) and decussated commissural axons frequently reenter/recross the FP in mice lacking all three Slits (Long et al., 2004). Notably, midline recrossing events were also not observed in Robo 1 and Robo2 single knock-out mice (Long et al., 2004), raising the possibility that these Robos function redundantly to bar the reentry of decussated commissural axons into the FP. In an attempt to address this issue and to reconcile the results of our misexpression experiments with the phenotype of the Slit triple-mutant mouse, we performed shRNA interference experiments aimed at knocking down Robol and Robo 2 in chick embryos. Unfortunately, we were only able to achieve a partial knockdown of robol mRNA and this did not perturb commissural axon pathfinding (see Materials and Methods) (supplemental Fig. 8, available at www.jneurosci.org as supplemental material). 
Table 1. In embryos coelectroporated with Neurog1tauGFP and CMV-Robo2FL-myc (Rb2), compared with embryos electroporated with Neurog1tauGFP (WT), more axons turn into the longitudinal plan on the ipsilateral side of the FP

\begin{tabular}{lccccc}
\hline Groups & WT mean & Rb2 mean & WT median & Rb2 median & Sample size \\
\hline Vertical objects & 8.25 & 19.45 & $8^{a *}$ & $16^{a *}$ & WT: 24 \\
Horizontal objects & 34.83 & 38.25 & $33.5^{a * *}$ & $32^{a * *}$ & Rb2: 31 \\
Ratio: vertical/horizontal & 0.24 & 0.51 & $0.24^{b_{* * *}}$ & $0.50^{b * * *}$ & Rb2: 8 \\
& & & & & WT: 24 \\
& Rb2 ratio/WT ratio: 2.13 & Rb2 ratio/WT ratio: 2.08 & \\
\hline
\end{tabular}

We compared the number of GFP-positive axons projecting into the longitudinal plane on the ipsilateral side of the FP in open-book preparations obtained from embryos electroporated with Neurog1tauGFP (WT) or coelectroporated with Neurog1tauGFP/CMV-Robo2FL-myc (Rb2). This was done by first counting the number of objects captured in the vertical line scans (which represent longitudinally projecting axons) and the number of objects captured in the horizontal line scans (which represent electroporated cell bodies). The ratios of vertical-to-horizontal objects were then calculated using the means and medians of these values for the two different groups. In all cases, object analysis was performed as described in Materials and Methods.

${ }^{a, b}$ Statistical significance of the medians and the median value ratios: ${ }^{a}$ two-tailed and ${ }^{b}$ one-tailed.

Highly significant: ${ }^{*} p=0.009,{ }^{* * *} p<0.0001$; not significant: ${ }^{* *} p=0.9497$

A very recent study has shown that Robo1/2 double knockout mice phenocopy the pathfinding defects we observe after misexpressing truncated Robos (Chen et al., 2008). Furthermore, a newly identified isoform of Robo3, Robo3.2, is selectively expressed on postcrossing commissural axons in the mouse spinal cord and these axons inappropriately reenter the FP after knockdown of Robo3.2 in Robo1/2 null embryos (Chen et al., 2008). Assuming that a similar situation exists in the chick spinal cord, this additional Slit receptor might have a higher affinity for Slit and/or requires a lower concentration of Slit than Robo1 or Robo2 to mediate a repulsive response at the ventral midline. Accordingly, misexpressed truncated Robos may bind enough Slit to effectively disable Robol and Robo2, but not a highly sensitive, third repulsive Robo, which may prevent midline recrossing in chick embryos. Alternatively, Robo receptors may be capable of binding other midline repellents, and/or other long- (e.g., Npn-Sema) (Zou et al., 2000) or short-range (EphB-ephrin-B) (Kadison et al., 2006) guidance systems may operate in parallel with Robo-Slit signaling to prevent decussated commissural axons from reentering/recrossing the floor plate. In principle, the genetic labeling strategy described here could be used in conjunction with loss-of-function and gain-of-function approaches to investigate the in vivo roles of these and other axon navigation systems within relatively homogeneous populations of commissural axons.

\section{References}

Briscoe J, Sussel L, Serup P, Hartigan-O'Connor D, Jessell TM, Rubenstein JL, Ericson J (1999) Homeobox gene Nkx2.2 and specification of neuronal identity by graded Sonic hedgehog signalling. Nature 398:622-627.

Brittis PA, Lu Q, Flanagan JG (2002) Axonal protein synthesis provides a mechanism for localized regulation at an intermediate target. Cell 110:223-235.

Charron F, Stein E, Jeong J, McMahon AP, Tessier-Lavigne M (2003) The morphogen Sonic Hedgehog is an axonal chemoattractant that collaborates with Netrin-1 in midline axon guidance. Cell 113:11-23.

Chen Z, Gore BB, Long H, Ma L, Tessier-Lavigne M (2008) Alternative splicing of the Robo3 axon guidance receptor governs the midline switch from attraction to repulsion. Neuron 58:325-332.

Chesnutt C, Niswander L (2004) Plasmid-based short-hairpin RNA interference in the chicken embryo. Genesis 39:73-78.

Dickson BJ, Gilestro GF (2006) Regulation of commissural axon pathfinding by Slit and its Robo receptors. Annu Rev Cell Dev Biol 22:651-675.

Dillon AK, Fujita SC, Matise MP, Jarjour AA, Kennedy TE, Kollmus H, Arnold HH, Weiner JA, Sanes JR, Kaprielian Z (2005) Molecular control of spinal accessory motor neuron/axon development in the mouse spinal cord. J Neurosci 25:10119-10130.

Gowan K, Helms AW, Hunsaker TL, Collisson T, Ebert PJ, Odom R, Johnson JE (2001) Crossinhibitory activities of Ngn1 and Math 1

allow specification of distinct dorsal interneurons. Neuron 31:219-232.

Hamburger V, Hamilton HL (1951) A series of normal stages in the development of the chick embryo. J Morphol 88:49-92.

Hammond R, Vivancos V, Naeem A, Chilton J, Mambetisaeva E, Mambitisaeva E, Andrews W, Sundaresan V, Guthrie S (2005) Slitmediated repulsion is a key regulator of motor axon pathfinding in the hindbrain. Development 132:4483-4495.

Imondi R, Kaprielian Z (2001) Commissural axon pathfinding on the contralateral side of the floor plate: a role for B-class ephrins in specifying the dorsoventral position of longitudinally-projecting commissural axons. Development 128: $4859-4871$.

Imondi R, Wideman C, Kaprielian Z (2000) Complementary expression of transmembrane ephrins and their receptors in the mouse spinal cord: a possible role in constraining the orientation of longitudinally projecting axons. Development 127:1397-1410.

Jevince AR, Kadison SR, Pittman AJ, Chien CB, Kaprielian Z (2006) Distribution of $\mathrm{EphB}$ receptors and ephrin-B1 in the developing vertebrate spinal cord. J Comp Neurol 497:734-750.

Jia L, Cheng L, Raper J (2005) Slit/Robo signaling is necessary to confine early neural crest cells to the ventral migratory pathway in the trunk. Dev Biol 282:411-421.

Kadison SR, Kaprielian Z (2004) Diversity of contralateral commissural projections in the embryonic rodent spinal cord. J Comp Neurol 472:411-422.

Kadison SR, Mäkinen T, Klein R, Henkemeyer M, Kaprielian Z (2006) EphB receptors and ephrin-B3 regulate axon guidance at the ventral midline of the embryonic mouse spinal cord. J Neurosci 26:8909-8914.

Kaprielian Z, Cho KO, Hadjiargyrou M, Patterson PH (1995) CD9, a major platelet cell surface glycoprotein, is a ROCA antigen and is expressed in the nervous system. J Neurosci 15:562-573.

Krull CE (2004) A primer on using in ovo electroporation to analyze gene function. Dev Dyn 229:433-439.

Long H, Sabatier C, Ma L, Plump A, Yuan W, Ornitz DM, Tamada A, Murakami F, Goodman CS, Tessier-Lavigne M (2004) Conserved roles for Slit and Robo proteins in midline commissural axon guidance. Neuron 42:213-223.

Lumpkin EA, Collisson T, Parab P, Omer-Abdalla A, Haeberle H, Chen P, Doetzlhofer A, White P, Groves A, Segil N, Johnson JE (2003) Math1driven GFP expression in the developing nervous system of transgenic mice. Gene Expr Patterns 3:389-395.

Mambetisaeva ET, Andrews W, Camurri L, Annan A, Sundaresan V (2005) Robo family of proteins exhibit differential expression in mouse spinal cord and Robo-Slit interaction is required for midline crossing in vertebrate spinal cord. Dev Dyn 233:41-51.

Nakada Y, Parab P, Simmons A, Omer-Abdalla A, Johnson JE (2004) Separable enhancer sequences regulate the expression of the neural bHLH transcription factor neurogenin-1. Dev Biol 271:479-487.

Ou SK-H, McDonald C, Patterson PH (1991) Comparison of two techniques for targeting the production of monoclonal antibodies against particular antigens. J Immunol Methods 145:111-118.

Sabatier C, Plump AS, Le Ma, Brose K, Tamada A, Murakami F, Lee EY, Tessier-Lavigne M (2004) The divergent Robo family protein Rig-1/ Robo3 is a negative regulator of Slit responsiveness required for midline crossing by commissural axons. Cell 117:157-169.

Serafini T, Colamarino SA, Leonardo ED, Wang H, Beddington R, Skarnes WC, Tessier-Lavigne M (1996) Netrin-1 is required for commissural axon guidance in the developing vertebrate nervous system. Cell 87:1001-1014. 
Shaner NC, Steinbach PA, Tsien RY (2005) A guide to choosing fluorescent proteins. Nat Methods 2:905-909.

Stein E, Tessier-Lavigne M (2001) Hierarchical organization of guidance receptors: silencing of Netrin attraction by Slit through a Robo/DCC receptor complex. Science 291:1928-1938.

Stoeckli ET, Landmesser LT (1995) Axonin-1, Nr-CAM, and Ng-CAM play different roles in the in vivo guidance of chick commissural neurons. Neuron 14:1165-1179.

Timmer J, Johnson J, Niswander L (2001) The use of in ovo electroporation for the rapid analysis of neural-specific murine enhancers. Genesis 29:123-132.

Vargesson N, Luria V, Messina I, Erskine L, Laufer E (2001) Expression patterns of Slit and Robo family members during vertebrate limb development. Mech Dev 106:175-180.
Weiner JA, Koo SJ, Nicolas S, Fraboulet S, Pfaff SL, Pourquie O, Sanes JR (2004) Axon fasciculation defects and retinal dysplasias in mice lacking the immunoglobulin superfamily adhesion molecule BEN/ALCAM/SC1. Mol Cell Neurosci 27:59-69.

Whitford KL, Marillat V, Stein E, Goodman CS, Tessier-Lavigne M, Chédotal A, Ghosh A (2002) Regulation of cortical dendrite development by SlitRobo interactions. Neuron 33:47-61.

Yee SP, Rigby PW (1993) The regulation of myogenin gene expression during the embryonic development of the mouse. Genes Dev 7:1277-1289.

Zou Y, Stoeckli E, Chen H, Tessier-Lavigne M (2000) Squeezing axons out of the gray matter: a role for Slit and Semaphorin proteins from midline and ventral spinal cord. Cell 102:363-375. 